\title{
AN EXAMPLE IN ČECH COHOMOLOGY
}

\author{
DANIEL S. KAHN ${ }^{1}$
}

In this note, we give an example of a compact space $X$ with integral Cech cohomology groups $H^{q}(X)=0, q>0$, but which can be mapped essentially onto the three-sphere $S^{3}$. This cannot occur for finitedimensional $X[2]$.

We construct such an $X$ for each odd prime $p$, which we now suppose fixed. Define $X_{0}$ to be $S^{2 p} \bigcup_{p} e^{2 p+1}$, the $2 p$-sphere with a $(2 p+1)$ cell attached by a map of degree $p$. Inductively, we define $X_{n+1}$ to be the (2p-2)-fold suspension $E^{2 p-2} X_{n}$ of $X_{n}, n \geqq 0$. We also define maps $\alpha_{n}: X_{n} \rightarrow X_{n-1}, n>1$, by $\alpha_{n}=E^{2 p-2} \alpha_{n-1}$, where $\alpha_{1}$ is defined as follows: Let $\beta: S^{2 p} \rightarrow S^{3}$ represent a generator of $\pi_{2 p}\left(S^{3} ; p\right) \approx Z_{p}$. Then $E^{2 p-3} \beta: S^{4 p-3} \rightarrow S^{2 p}$ admits a coextension $\beta^{\prime}: S^{4 p-2} \rightarrow S^{2 p} \bigcup_{p} e^{2 p+1}[4$, p. 13]. Since the homotopy class of $\beta^{\prime}$ is of order $p, \beta^{\prime}$ admits an extension $\alpha_{1}: S^{4 p-2} \bigcup_{p} e^{4 p-1} \rightarrow S^{4 p-1} \bigcup_{p} e^{2 p+1}$. We note that $\beta$ admits an extension $\alpha: S^{2 p} \bigcup_{p} e^{2 p+1} \rightarrow S^{3}$. We now define $X=\operatorname{Inv} \operatorname{Lim}\left\{X_{n}, \alpha_{n}\right\}$. It is evident that $H^{q}(X)=0, q>0$. The composites $f_{n}=\alpha \alpha_{1} \alpha_{2} \cdots \alpha_{n}$ : $X_{n} \rightarrow S^{3}$ define a map $f: X \rightarrow S^{3}$. The proof that $f$ is essential depends on the following result of Toda [5], [1].

THEOREM [TODA]. Each $f_{n}$ is an essential map. Further, all suspensions of $f_{n}$ are essential.

Since $\left[X, S^{3}\right]$, the set of homotopy classes of maps of $X \rightarrow S^{3}$, is equal to $\operatorname{Dir} \operatorname{Lim}\left\{\left[X_{n}, S^{3}\right], \alpha_{n+1}^{*}\right\}$ [3, p. 228], $f$ is essential.

$X$ has the further property that $E^{n(2 p-2)} X=X, n>0$. The theorem of Toda implies that each $E^{n(2 p-2)} f: E^{n(2 p-2)} X=X \rightarrow S^{3+n(2 p-2)}$ is also essential.

\section{BIBLIOGRAPHY}

1. J. F. Adams, Lectures on $K^{*}(X)$, Mimeographed notes, University of Manchester, 1962.

2. S. T. Hu, Mappings of a normal space into an absolute neighborhood retract, Trans. Amer. Math. Soc. 64 (1948), 336-358.

3. E. Spanier, Borsuk's cohomotopy groups, Ann. of Math. 50 (1949), 203-245.

4. H. Toda, Composition methods in homotopy groups of spheres, Princeton Univ. Press, Princeton, N. J., 1962.

5. - On unstable homotopy of spheres and classical groups, Proc. Nat. Acad. Sci. 46 (1960), 1102-1105.

The University of Chicago

Received by the editors May 15, 1964.

${ }^{1}$ This research was partially supported by National Science Foundation Grant GP-623. 